\title{
WAVE PROPAGATION IN PIEZOELECTRIC RINGS WITH RECTANGULAR CROSS-SECTIONS
}

\author{
XIAOMING Zhang \\ School of Mechanical and Power Engineering, Henan Polytechnic University, Jiaozuo, P.R. China \\ e-mail: zxmworld11@163.com \\ YOUCHAO WANG \\ School of Materials Science and Engineering, Henan Polytechnic University, Jiaozuo, P.R. China \\ e-mail:wangyc@hpu.edu.cn \\ Huitao Chen \\ School of Mechanical and Power Engineering, Henan Polytechnic University, Jiaozuo, P.R. China \\ e-mail: huitaochen@hpu.edu.cn
}

\begin{abstract}
The ring ultrasonic transducers are widely used in the ocean engineering and medical fields. This paper employs an extended orthogonal polynomial approach to solve the guided wave propagation in two-dimensional structures, i.e. piezoelectric rings with rectangular cross-sections. The extended polynomial approach can overcome the drawbacks of the conventional orthogonal polynomial approach which can be used to solve wave propagation in one-dimensional structures. Through numerical comparison with the available results for a rectangular aluminum bar, the validity of the present approach is illustrated. The dispersion curves and displacement and electric potential distributions of various rectangular piezoelectric rings are calculated, and the effects of different radius to thickness ratios, width to height ratios and polarizing directions on the dispersion curves are illustrated.
\end{abstract}

Keywords: piezoelectric rings, orthogonal polynomial, guided wave, dispersion curves

\section{Introduction}

With the development of materials and advances in manufacturing technology, piezoelectric materials having electromechanical coupling effects have found extensive applications in many smart devices, and the behavior of the selected wave mode can directly affect the performance of the devices. Thus, it is very important to study the wave characteristics in piezoelectric structures. Much effort has been made both theoretically and experimentally to study wave propagation in piezoelectric structures by scientists and engineers, which is essential for the application of piezoelectric materials. Wave propagation along piezoelectric cylindrical rods of hexagonal 6, 622, and $6 \mathrm{~mm}$ crystal symmetry was discussed by Wilson and Morrison (1977). The propagating nature of the elastic and electric wave in bone and porous PZT was investigated by Chakraborty (2009). Using the extended Durbin method, Ing et al. (2013) investigated the transient elastic waves propagating in a two-layered piezoelectric medium The wave propagation behavior in layered piezoelectric structures and functionally graded piezoelectric material structures has also been studied by many researches with different methods, such as the transfer matrix method (Cai et al., 2001), the layer element method (Han et al., 2004), the orthogonal polynomial series method (Yu and Ma, 2008; Yu et al., 2013; Singh and Rokne, 2013), and so on.

As a common structure, hollow cylinder has been paid considerable attention on the wave propagation. For piezoelectric media, Paul and Venkatesan (1987) and Shul'ga (2002) studied three dimensional electroelastic waves and the axisymmetric waves in a hollow piezoelectric 
ceramic cylinder. Puzyrev and Storozhev (2011) studied the problem of electroelastic waves propagating in piezoelectric hollow cylinders of sector cross section and analyzed mode asymptotic behavior and amplitude distributions of wave characteristics. Zenkour (2012) developed an analytical solution to the axisymmetric problem of a radially polarized piezoelectric hollow cylinders subjected to electric, thermal and mechanical load. Using the Legendre orthogonal polynomial approach developed by Lefebvre et al. (1999) to solve the waves in multilayered plates, Yu et al. (2009) investigated the wave characteristics in functionally graded piezoelectric hollow cylinders. These investigations focused on one-dimensional structures, i.e. the hollow cylinders are infinite in axial direction and have a finite dimension in only one direction. But in practical applications, many piezoelectric elements have very finite dimensions in two directions. One-dimensional models are not suitable for these structures. Thus, it is significant to study the wave characteristics in 2-D piezoelectric structures, such as a piezoelectric ring with rectangular cross-section. The ring ultrasonic transducer has been widely used in ocean engineering and medical fields. But few investigations on the wave propagation in ring transducers have been reported.

In this paper, we present an extended orthogonal polynomial series approach to solve the wave propagation in a 2-D structure, i.e. a piezoelectric ring with rectangular cross-section. The present approach can overcome the drawbacks of the conventional orthogonal polynomial approach which can only deal with the one-dimensional structures that have a finite dimension in only one direction, such as the axially infinite hollow cylinder, horizontally infinite flat plate. The dispersion curves and displacement and electric potential distributions of various piezoelectric rings with rectangular cross sections are shown. The effects of different width to height ratios, radius to thickness ratios and polarizing directions on the wave characteristics are also illustrated. The investigating results can be used to direct the design and optimization of the piezoelectric ring transducers.

\section{Mathematics and formulation of the problem}

In this seciton, we derive the analytical formulation of the problem in cylindrical coordinate $(r, \theta, z)$ with the $z$-axis coinciding with the axis of the ring. Considering an orthotropic ring with rectangular cross-section, $h$ is height in $\mathrm{z}$ direction and $a, b$ denote the inner and outer radius respectively, as shown in Fig. 1. The radius to thickness ratio is defined as $\eta=b /(b-a)$ and the width to height ratio is $d / h$. The polarizing direction is in $r$ or $z$ direction. In this paper, traction free and open circuit boundary conditions are assumed.

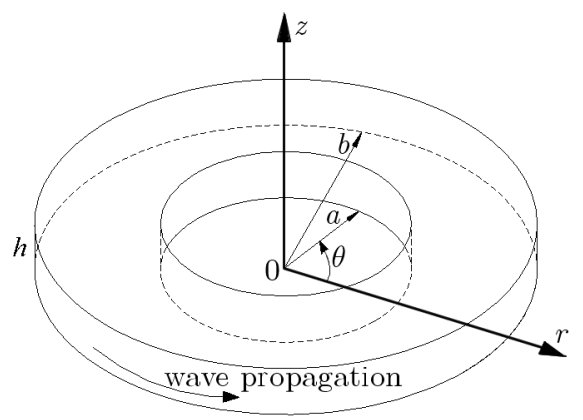

Fig. 1. Schematic of a piezoelectric ring with rectangular cross-section

In cylindrical coordinate, the dynamic equation for the piezoelectric ring is governed by

$$
\begin{aligned}
& \frac{\partial T_{r r}}{\partial r}+\frac{1}{r} \frac{\partial T_{r \theta}}{\partial \theta}+\frac{\partial T_{r z}}{\partial z}+\frac{T_{r r}-T_{\theta \theta}}{r}=\rho \frac{\partial^{2} u_{r}}{\partial t^{2}} \\
& \frac{\partial T_{r \theta}}{\partial r}+\frac{1}{r} \frac{\partial T_{\theta \theta}}{\partial \theta}+\frac{\partial T_{\theta z}}{\partial z}+\frac{2 T_{r \theta}}{r}=\rho \frac{\partial^{2} u_{\theta}}{\partial t^{2}}
\end{aligned}
$$




$$
\begin{aligned}
& \frac{\partial T_{r z}}{\partial r}+\frac{1}{r} \frac{\partial T_{\theta z}}{\partial \theta}+\frac{\partial T_{z z}}{\partial z}+\frac{T_{r z}}{r}=\rho \frac{\partial^{2} u_{z}}{\partial t^{2}} \\
& \frac{\partial D_{r}}{\partial r}+\frac{1}{r} \frac{\partial D_{\theta}}{\partial \theta}+\frac{\partial D_{z}}{\partial z}+\frac{D_{r}}{r}=0
\end{aligned}
$$

where $u_{i}, T_{i j}$ and $D_{i}$ denote mechanical displacement, the stress and electric displacement components respectively; $\rho$ is the density of the material.

The strain-displacement relations are

$$
\begin{aligned}
& \varepsilon_{r r}=\frac{\partial u_{r}}{\partial r} \quad \varepsilon_{\theta \theta}=\frac{1}{r} \frac{\partial u_{\theta}}{\partial \theta}+\frac{u_{r}}{r} \quad \varepsilon_{z z}=\frac{\partial u_{z}}{\partial z} \\
& \varepsilon_{\theta z}=\frac{1}{2}\left(\frac{\partial u_{\theta}}{\partial z}+\frac{\partial u_{z}}{r \partial \theta}\right) \quad \varepsilon_{r z}=\frac{1}{2}\left(\frac{\partial u_{r}}{\partial z}+\frac{\partial u_{z}}{\partial r}\right) \quad \varepsilon_{r \theta}=\frac{1}{2}\left(\frac{1}{r} \frac{\partial u_{r}}{\partial \theta}+\frac{\partial u_{\theta}}{\partial r}-\frac{u_{\theta}^{(2.2)}}{r}\right)
\end{aligned}
$$

where $\varepsilon_{i j}$ denotes the strain.

The constitutive equations for the piezoelectric ring with radial polarizing direction can be written in the following form

$$
\begin{aligned}
T_{\theta \theta} & =C_{11} \varepsilon_{\theta \theta}+C_{12} \varepsilon_{z z}+C_{13} \varepsilon_{r r}+e_{31} \frac{\partial \Phi}{\partial r} \\
T_{z z} & =\left(C_{12} \varepsilon_{\theta \theta}+C_{22} \varepsilon_{z z}+C_{23} \varepsilon_{r r}+e_{32} \frac{\partial \Phi}{\partial r}\right) \mathrm{I}(r, z) \\
T_{r r} & =\left(C_{13} \varepsilon_{\theta \theta}+C_{23} \varepsilon_{z z}+C_{33} \varepsilon_{r r}+e_{33} \frac{\partial \Phi}{\partial r}\right) \mathrm{I}(r, z) \\
T_{r z} & =\left(2 C_{44} \varepsilon_{r z}+e_{24} \frac{\partial \Phi}{\partial z}\right) \mathrm{I}(r, z) \\
T_{r \theta} & =\left(2 C_{55} \varepsilon_{r \theta}+e_{15} \frac{1}{r} \frac{\partial \Phi}{\partial \theta}\right) \mathrm{I}(r, z) \\
T_{\theta z} & =2 C_{66} \varepsilon_{\theta z} \mathrm{I}(r, z)
\end{aligned}
$$

and

$$
\begin{aligned}
& D_{\theta}=2 e_{15} \varepsilon_{r \theta}-\epsilon_{11} \frac{1}{r} \frac{\partial \Phi}{\partial \theta} \\
& D_{z}=\left(2 e_{24} \varepsilon_{r z}-\epsilon_{22} \frac{\partial \Phi}{\partial z}\right) \mathrm{I}(r, z) \\
& D_{r}=\left(e_{31} \varepsilon_{\theta \theta}+e_{32} \varepsilon_{z z}+e_{33} \varepsilon_{r r}-\epsilon_{33} \frac{\partial \Phi}{\partial r}\right) \mathrm{I}(r, z)
\end{aligned}
$$

where $\Phi$ denotes the electric potential. $C_{i j}, e_{i j}$ and $\epsilon_{i j}$ are the elastic, piezoelectric and dielectric coefficients given in the crystallographic axes, respectively. $\mathrm{I}(r, z)$ is the rectangular window function, introduced so as to meet the stress-free boundary conditions $\left(T_{r r}=T_{r \theta}=T_{r z}=T_{\theta z}=\right.$ $T_{z z}=D_{r}=D_{z}=0$ at the four boundaries), defined as

$$
\mathrm{I}(y, z)=\left\{\begin{array}{ll}
1 & 0 \leqslant y \leqslant d \\
0 & \text { elsewhere }
\end{array} \text { and } 0 \leqslant z \leqslant h\right.
$$

For a free harmonic plane wave propagating in the circumferential direction in a ring, we assume the displacement components, to be of the form

$$
\begin{array}{ll}
u_{r}(r, \theta, z, t)=\exp (\mathrm{i} k b \theta-\mathrm{i} \omega t) U(r, z) & u_{\theta}(r, \theta, z, t)=\exp (\mathrm{i} k b \theta-\mathrm{i} \omega t) V(r, z) \\
u_{z}(r, \theta, z, t)=\exp (\mathrm{i} k b \theta-\mathrm{i} \omega t) W(r, z) & \varphi(r, \theta, z, t)=\exp (\mathrm{i} k b \theta-\mathrm{i} \omega t) X(r, z)
\end{array}
$$

where $U(r, z), V(r, z)$ and $W(r, z)$ denote the mechanical displacement amplitudes in the radial, circumferential and axial directions respectively, and $X(r, z)$ represents the amplitude of electric potential. $\omega$ is the angular frequency, and $k$ is the magnitude of the wave vector. 
Substituting Eqs. (2.2)-(2.6) into Eq. (2.1), the governing differential equations in terms of mechanical displacement and electric potential components, gives

$$
\begin{aligned}
& {\left[C_{33}\left(r^{2} U_{, r r}+r U_{, r}\right)-C_{11} U-k^{2} b^{2} C_{55} U+C_{44} r^{2} U_{, z z}-\mathrm{i} k b\left(C_{11}+C_{55}\right) V+\left(C_{23}-C_{12}\right) r W,_{z}\right.} \\
& +\mathrm{i} k b\left(C_{13}+C_{55}\right) r V_{, r}+\left(C_{23}+C_{44}\right) r^{2} W_{,_{r z}}+e_{33}\left(r^{2} X_{, r r}+r X_{,_{r}}\right)-e_{31} r X_{,_{r}}-k^{2} b^{2} e_{15} X \\
& \left.+e_{24} r^{2} X, z z\right] I(r, z)+\left[C_{33} r^{2} U_{, r}+C_{13} r(\mathrm{i} k b V+U)+C_{23} r^{2} W,_{z}+e_{33} r^{2} X,_{r}\right] I(r, z){ }_{, r} \\
& +\left[C_{44} r^{2}\left(U, z+W,_{r}\right)+e_{24} r^{2} X,,_{z}\right] I(r, z)_{, z}=-\rho r^{2} \omega^{2} U \\
& {\left[C_{55}\left(r^{2} V_{, r r}+r V_{, r}\right)-\left(C_{55}+(k b)^{2} C_{11}\right) V+\mathrm{i} k b\left(C_{13}+C_{55}\right) r U_{, r}+C_{66} r^{2} V_{, z z}\right.} \\
& \left.+\mathrm{i} k b\left(C_{11}+C_{66}\right) U+\mathrm{i} k b\left(C_{12}+C_{66}\right) r W_{, z}+\left(e_{31}+e_{15}\right) r X_{, r}+2 e_{15} X\right] I(r, z) \\
& +C_{66}\left(r^{2} V,_{z}+\mathrm{i} k b r W\right) I(r, z){ }_{, z}+\left[C_{55}\left(r^{2} V_{, r}+r V+\mathrm{i} k b r U\right)+e_{15} r X\right] I(r, z){ }_{, r}=-\rho r^{2} \omega^{2} V \\
& {\left[C_{44}\left(r^{2} W,_{r r}+r W,_{r}\right)+C_{22} r^{2} W_{, z z}-(k b)^{2} C_{66} W+\left(C_{12}+C_{44}\right) r U_{, z}+\left(C_{23}+C_{44}\right) r^{2} U_{, r z}(2.7)\right.} \\
& \left.+\mathrm{i} k b\left(C_{12}+C_{66}\right) r V_{, z}+e_{24} r X_{, z}+\left(e_{24}+e_{32}\right) r^{2} X_{, r z}\right] I(r, z)+\left[C_{12} r(\mathrm{i} k b V+U)\right. \\
& \left.+C_{23} r^{2} U_{, r}+C_{22} r^{2} W_{, z}+e_{32} r^{2} X, r\right] I(r, z)_{, z}+\left[C_{44} r^{2}\left(W_{, r}+U_{, z}\right)+e_{24} r^{2} X, z\right] I(r, z)_{, r} \\
& =-\rho r^{2} \omega^{2} V \\
& {\left[e_{33}\left(r^{2} U_{, r r}+r U_{,_{r}}\right)-k^{2} b^{2} e_{15} U+e_{31} r U_{,_{r}}+e_{24} r^{2} U_{, z z}+\left(e_{31}+e_{15}\right) r V_{,_{r}}-e_{15} V\right.} \\
& \left.+\left(e_{24}+e_{32}\right) r^{2} W_{, r z}+e_{24} r W_{, z}-\epsilon_{33}\left(r^{2} X, r r+r X, r\right)-\epsilon_{22} r^{2} X, z z+(k b)^{2} \epsilon_{11} X\right] I(r, z) \\
& +\left[e_{24} r^{2} U_{, z}+e_{24} r^{2} W_{, r}-\epsilon_{22} r^{2} X, z\right] I(r, z), z \\
& +\left[e_{31} r U+e_{33} r^{2} U_{, r}+e_{31} r V+e_{32} r^{2} W_{, z}-\epsilon_{33} r^{2} X,{ }_{r}\right] I(r, z){ }_{, r}=0
\end{aligned}
$$

where subscript comma indicates partial derivative.

To solve the coupled wave equation, we expand $U(r, z), V(r, z), W(r, z)$ and $X(r, z)$ into products of two Legendre orthogonal polynomial series

$$
\begin{array}{ll}
U(r, z)=\sum_{m, j=0}^{\infty} p_{m, j}^{1} Q_{m}(r) Q_{j}(z) & V(r, z)=\sum_{m, j=0}^{\infty} p_{m, j}^{2} Q_{m}(r) Q_{j}(z) \\
W(r, z)=\sum_{m, j=0}^{\infty} p_{m, j}^{3} Q_{m}(r) Q_{j}(z) & X(r, z)=\sum_{m, j=0}^{\infty} p_{m, j}^{4} Q_{m}(r) Q_{j}(z)
\end{array}
$$

where $p_{m, j}^{i}(i=1,2,3,4)$ is the expansion coefficients and

$$
Q_{m}(r)=\sqrt{\frac{2 m+1}{b-a}} P_{m}\left(\frac{2 r-b-a}{b-a}\right) \quad Q_{n}(z)=\sqrt{\frac{2 n+1}{h}} P_{n}\left(\frac{2 z-h}{h}\right)
$$

with $P_{m}$ and $P_{n}$ representing the mth and the nth Legendre polynomial. The summation over the polynomials can be halted at some finite value $m=M$ and $n=N$, when higher order terms become essentially negligible.

Multiplying each equation by $Q_{j}(r) Q_{l}(z) \mathrm{e}^{-j \omega t}$ with $j$ and $l$ running respectively from zero to $M$ and zero to $N$, and integrating over $z$ from zero to $h$ and $r$ from $a$ to $b$ and taking advantage of the orthonormality of the polynomials $Q_{m}(r)$ and $Q_{n}(z)$, Eqs. (2.7) can be reorganized into a form of the system problem

$$
\begin{aligned}
& A_{11}^{j l m n} p_{m, n}^{1}+A_{12}^{j l m n} p_{m, n}^{2}+A_{13}^{j l m n} p_{m, n}^{3}+A_{14}^{j l m n} p_{m, n}^{4}=-\omega^{2} M_{j l m n} p_{m, n}^{1} \\
& A_{21}^{j l m n} p_{m, n}^{1}+A_{22}^{j l m n} p_{m, n}^{2}+A_{23}^{j l m n} p_{m, n}^{3}+A_{24}^{j l m n} p_{m, n}^{4}=-\omega^{2} M_{j l m n} p_{m, n}^{2} \\
& A_{31}^{j l m n} p_{m, n}^{1}+A_{32}^{j l m n} p_{m, n}^{2}+A_{33}^{j l m n} p_{m, n}^{3}+A_{34}^{j l m n} p_{m, n}^{4}=-\omega^{2} M_{j l m n} p_{m, n}^{3} \\
& A_{41}^{j l m n} p_{m, n}^{1}+A_{42}^{j l m n} p_{m, n}^{2}+A_{43}^{j l m n} p_{m, n}^{3}+A_{44}^{j l m n} p_{m, n}^{4}=0
\end{aligned}
$$


where $A_{\alpha \beta}^{j l m n}(\alpha, \beta=1,2,3,4)$ and $M_{j l m n}$ are the elements of a non-symmetric matrix.

Equations $(2.10)_{4}$ can be written as

$$
p_{m, n}^{4}=-\left(A_{44}^{j l m n}\right)^{-1}\left(A_{41}^{j l m n} p_{m, n}^{1}+A_{42}^{j l m n} p_{m, n}^{2}+A_{43}^{j l m n} p_{m, n}^{3}\right)
$$

Substituting Equation (2.11) into equations $(2.10)_{1},(2.10)_{2}$ and $(2.10)_{3}$, gives

$$
\begin{aligned}
& {\left[A_{11}^{j l m n}-A_{14}^{j l m n}\left(A_{44}^{j l m n}\right)^{-1} A_{41}^{j l m n}\right] p_{m, n}^{1}+\left[A_{12}^{j l m n}-A_{14}^{j l m n}\left(A_{44}^{j l m n}\right)^{-1} A_{42}^{j l m n}\right] p_{m, n}^{2}} \\
& +\left[A_{13}^{j l m n}-A_{14}^{j l m n}\left(A_{44}^{j l m n}\right)^{-1} A_{43}^{j l m n}\right] p_{m, n}^{3}=-\omega^{2} M_{j l m n} p_{m, n}^{1} \\
& {\left[A_{21}^{j l m n}-A_{24}^{j l m n}\left(A_{44}^{n, m}\right)^{-1} A_{41}^{j l m n}\right] p_{m, n}^{1}+\left[A_{22}^{j l m n}-A_{24}^{j l m n}\left(A_{44}^{j l m n}\right)^{-1} A_{42}^{j l m n}\right] p_{m, n}^{2}} \\
& \quad+\left[A_{23}^{j l m n}-A_{24}^{j l m n}\left(A_{44}^{j l m n}\right)^{-1} A_{43}^{j l m n}\right] p_{m, n}^{3}=-\omega^{2} M_{j l m n} p_{m, n}^{1} \\
& {\left[A_{31}^{j l m n}-A_{34}^{j l m n}\left(A_{44}^{j l m n}\right)^{-1} A_{41}^{j l m n}\right] p_{m, n}^{1}+\left[A_{32}^{j l m n}-A_{34}^{j l m n}\left(A_{44}^{j l m n}\right)^{-1} A_{42}^{j l m n}\right] p_{m, n}^{2}} \\
& \quad+\left[A_{33}^{j l m n}-A_{34}^{j l m n}\left(A_{44}^{j l m n}\right)^{-1} A_{43}^{j l m n}\right] p_{m, n}^{3}=-\omega^{2} M_{j l m n} p_{m, n}^{1}
\end{aligned}
$$

Then, Eqs. (2.12) can be recognized into

$$
\left[\begin{array}{lll}
\bar{A}_{11}^{j l m n} & \bar{A}_{12}^{j l m n} & \bar{A}_{13}^{j l m n} \\
\bar{A}_{21}^{j l m n} & \bar{A}_{22}^{j l m n} & \bar{A}_{23}^{j l m n} \\
\bar{A}_{31}^{j l m n} & \bar{A}_{32}^{j l m n} & \bar{A}_{33}^{j l m n}
\end{array}\right]\left\{\begin{array}{l}
p_{m, n}^{1} \\
p_{m, n}^{2} \\
p_{m, n}^{3}
\end{array}\right\}=-\omega^{2}\left[\begin{array}{ccc}
M_{j l m n} & 0 & 0 \\
0 & M_{j l m n} & 0 \\
0 & 0 & M_{j l m n}
\end{array}\right]\left\{\begin{array}{l}
p_{m, n}^{1} \\
p_{m, n}^{2} \\
p_{m, n}^{3}
\end{array}\right\}
$$

So, Eq. (2.13) forms the eigenvalue problem to be solved. The eigenvectors $p_{m, n}^{i}(i=1,2,3)$ allow the components of the displacement and $p_{m, n}^{4}$ determines the electric potential distribution. The eigenvalue $\omega^{2}$ gives angular frequency.

\section{Numerical results}

The computer programs in terms of the extended orthogonal polynomial approach have been written using Mathematica to calculate the dispersion curves and displacement and electric potential distributions for the piezoelectric rings. The physical properties of the piezoelectric material, PZT-4, are listed in Table 1. Here, the elastic constants of the radial polarizing ring and axial polarizing rings are the same to have a clear comparison.

Table 1. Material parameters of the piezoelectric materials

\begin{tabular}{|c|c|c|c|c|c|c|c|c|c|}
\hline Property & $C_{11}$ & $C_{12}$ & $C_{13}$ & $C_{22}$ & $C_{23}$ & $C_{33}$ & $C_{44}$ & $C_{55}$ & $C_{66}$ \\
\hline \hline \multirow{3}{*}{ r-polarization } & 13.9 & 7.8 & 7.4 & 13.9 & 7.4 & 11.5 & 2.56 & 2.56 & 3.05 \\
\cline { 2 - 10 } & $e_{15}$ & $e_{24}$ & $e_{31}$ & $e_{32}$ & $e_{33}$ & $\epsilon_{11}$ & $\epsilon_{22}$ & $\epsilon_{33}$ & $\rho$ \\
\cline { 2 - 10 } & 12.7 & 12.7 & -5.2 & -5.2 & 15.1 & 650 & 650 & 560 & 7.5 \\
\hline \multirow{2}{*}{ a-polarization } & $e_{34}$ & $e_{16}$ & $e_{23}$ & $e_{21}$ & $e_{22}$ & $\epsilon_{11}$ & $\epsilon_{22}$ & $\epsilon_{33}$ & $\rho$ \\
\cline { 2 - 9 } & 12.7 & 12.7 & -5.2 & -5.2 & 15.1 & 650 & 560 & 650 & 7.5 \\
\hline
\end{tabular}




\subsection{Approach validation}

To the authors' knowledge, there are not published results on the wave propagation for a piezoelectric ring with rectangular cross-section so far. In order to check the effectiveness of the present approach and validate the computer program, we calculate a $16 \mathrm{~mm}$ by $5 \mathrm{~mm}$ rectangular aluminum bar and make a comparison with previous results. The material parameters are $\rho=2.7 \cdot 10^{3} \mathrm{~kg} / \mathrm{m}^{3}, C_{11}=C_{22}=C_{33}=10.78 \cdot 10^{10} \mathrm{~Pa}, C_{12}=C_{13}=C_{23}=5.494 \cdot 10^{10} \mathrm{~Pa}$, $C_{44}=C_{55}=C_{66}=2.645 \cdot 10^{10} \mathrm{~Pa}$. Figure 2 is the corresponding dispersion curves, of which lines are from Loveday (2006), and dotted lines are obtained from the present approach. As can be seen, the agreement between the present approach and the previous results is quite good.

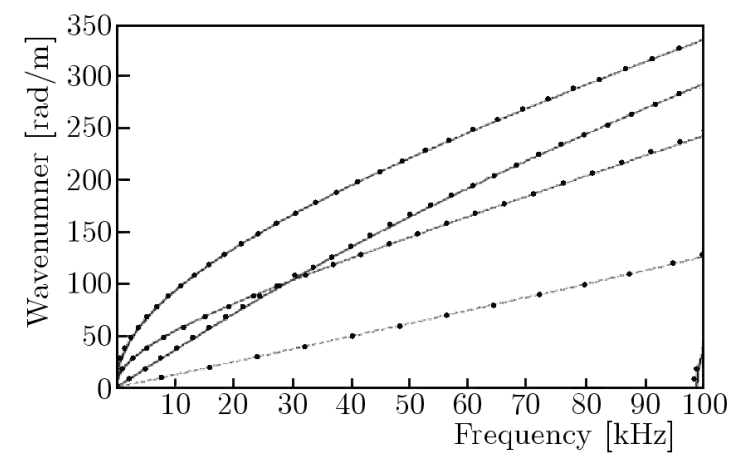

Fig. 2. Dispersion of propagating waves in a rectangular waveguide; lines: Philip W. Loveday's results, dotted lines: the authors' results

\subsection{Guided waves in piezoelectric rings with rectangular cross-sections}

Figure 3 shows the dispersion curves of the first four order modes for the PZT-4 ring with a square cross section and for the corresponding non-piezoelectric one with $h=1 \mathrm{~mm}, a=9 \mathrm{~mm}$, $b=10 \mathrm{~mm}$ and $\eta=10$. It can be seen that piezoelectricity has a significant effect on the dispersion curves. For any one specific mode, the phase velocities of non-piezoelectric ring are smaller than those of the corresponding piezoelectric one, and the piezoelectric effect is very little on the low order modes at low frequency and becomes stronger as the wave number and mode order increase. The wave number is usually very big and the operating frequency is very high in micro-scale SAW devices. So, the piezoelectric effects will be prominent.
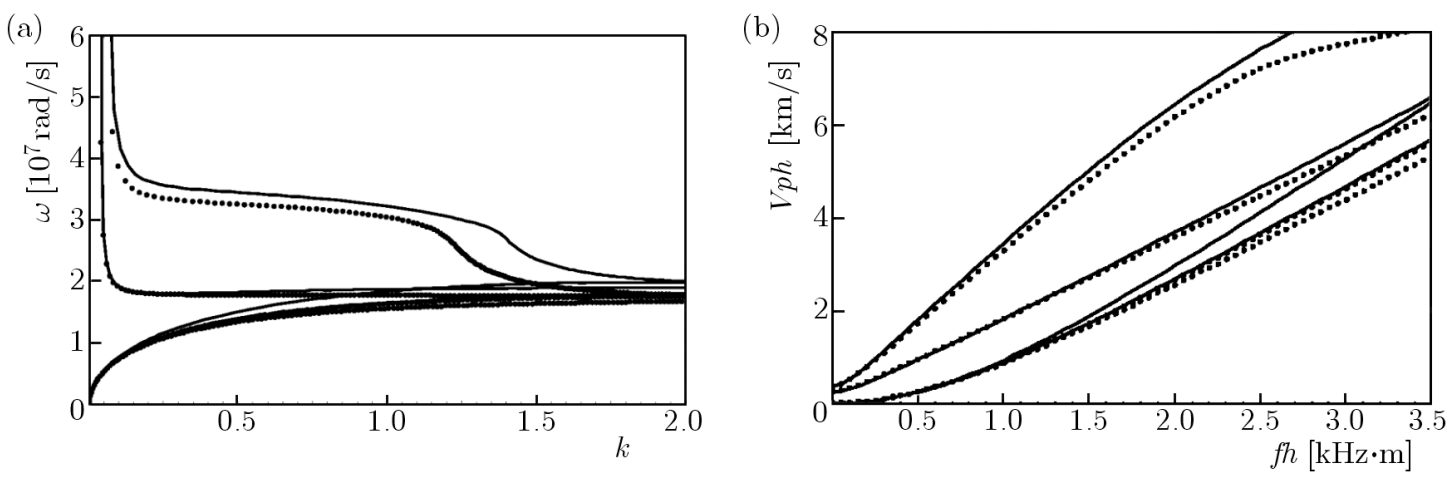

Fig. 3. Dispersion curves of the first four order modes for a square ring with $\eta=10$ : (a) phase velocity spectra, (b) frequency spectra, solid line, piezoelectric; dotted line, non-piezoelectric

Figures 4 and 5 show the dispersion curves of the PZT-4 rings with different radius to thickness ratios $(\eta=10, \eta=2)$ and different width to height ratios $(d / h=1 / 2, d / h=1 / 4$, $d / h=1 / 10)$, respectively. Figure 6 illustrates the dispersion curves of the PZT-4 ring with 
(a)

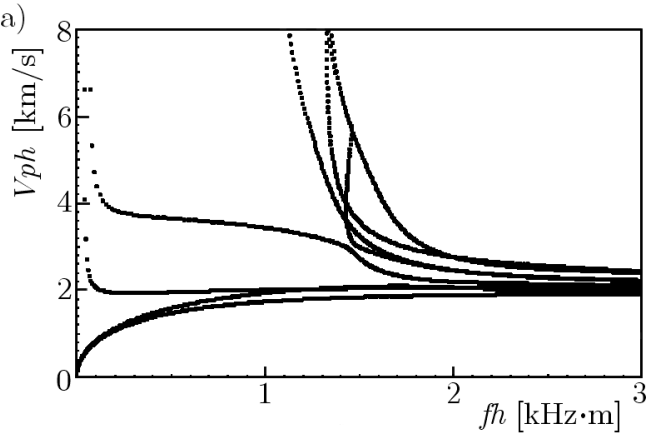

(b)

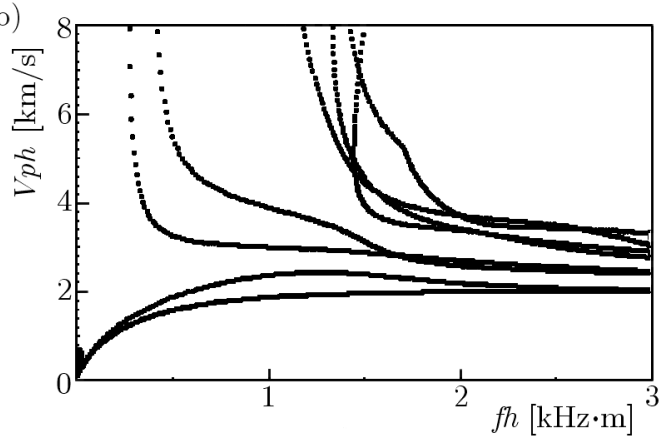

Fig. 4. Phase velocity dispersion curves for piezoelectric square rings: (a) $\eta=10$, (b) $\eta=2$
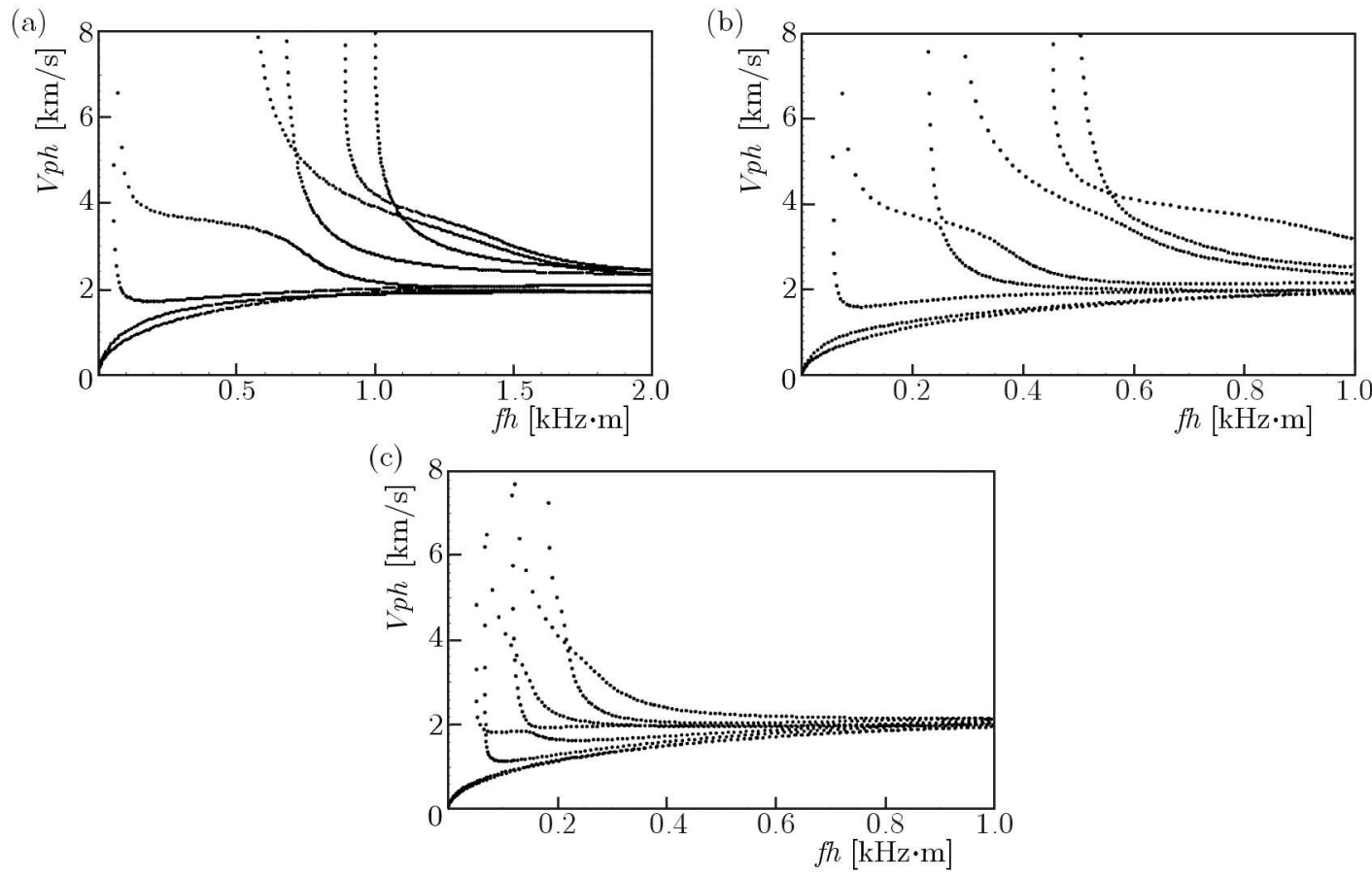

Fig. 5. Phase velocity dispersion curves for piezoelectric rectangular rings with different width to thickness ratios: (a) $d / h=1 / 2$, (b) $d / h=1 / 4$, (c) $d / h=1 / 10$

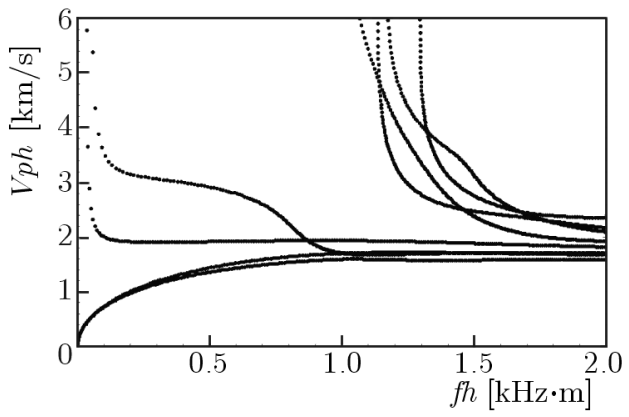

Fig. 6. Phase velocity dispersion curves for a piezoelectric rectangular ring with axial polarization 

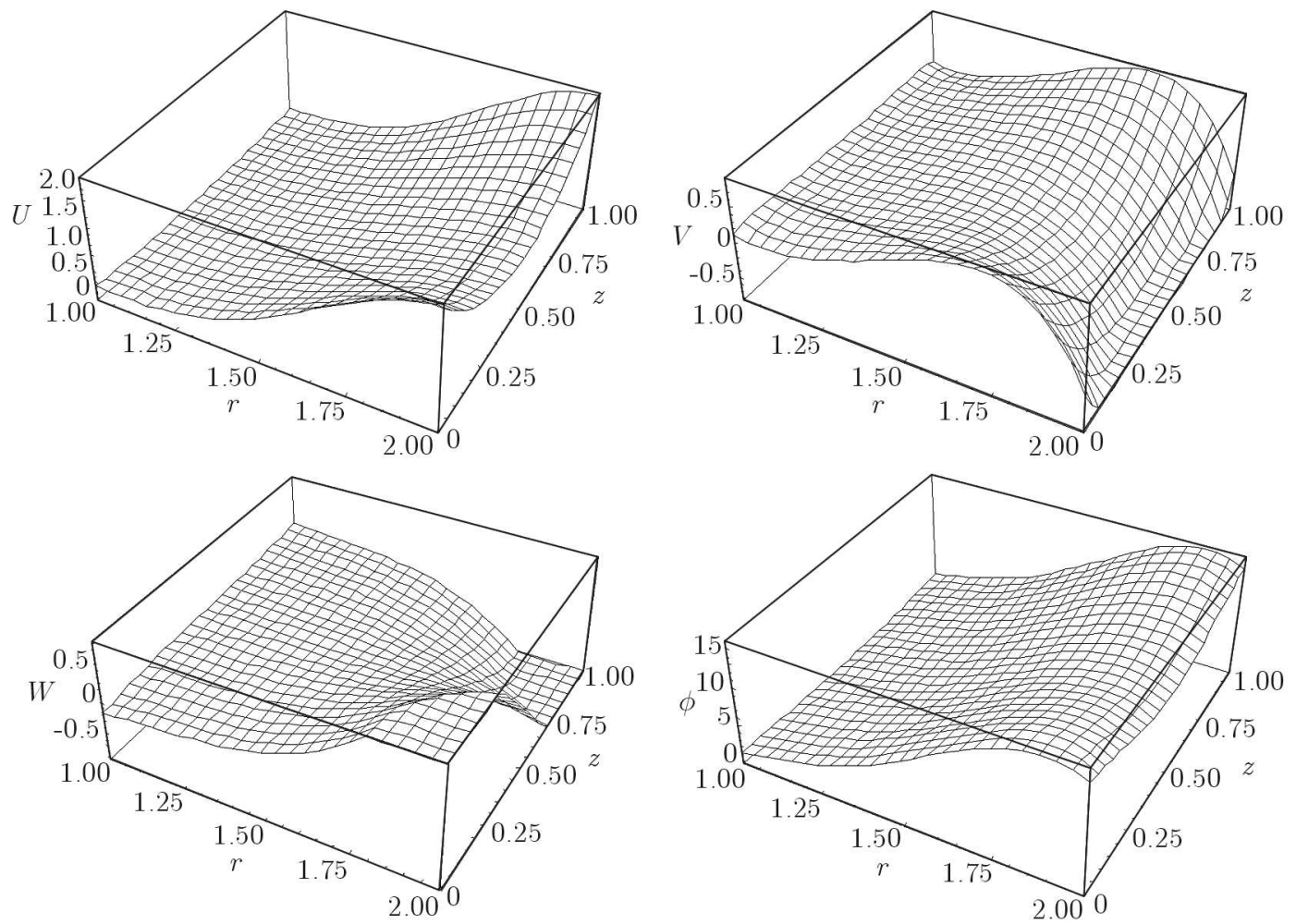

Fig. 7. Mechanical displacement and electric potential profiles of the first mode for a piezoelectric square ring with $\eta=2$ at $k d=4.1$
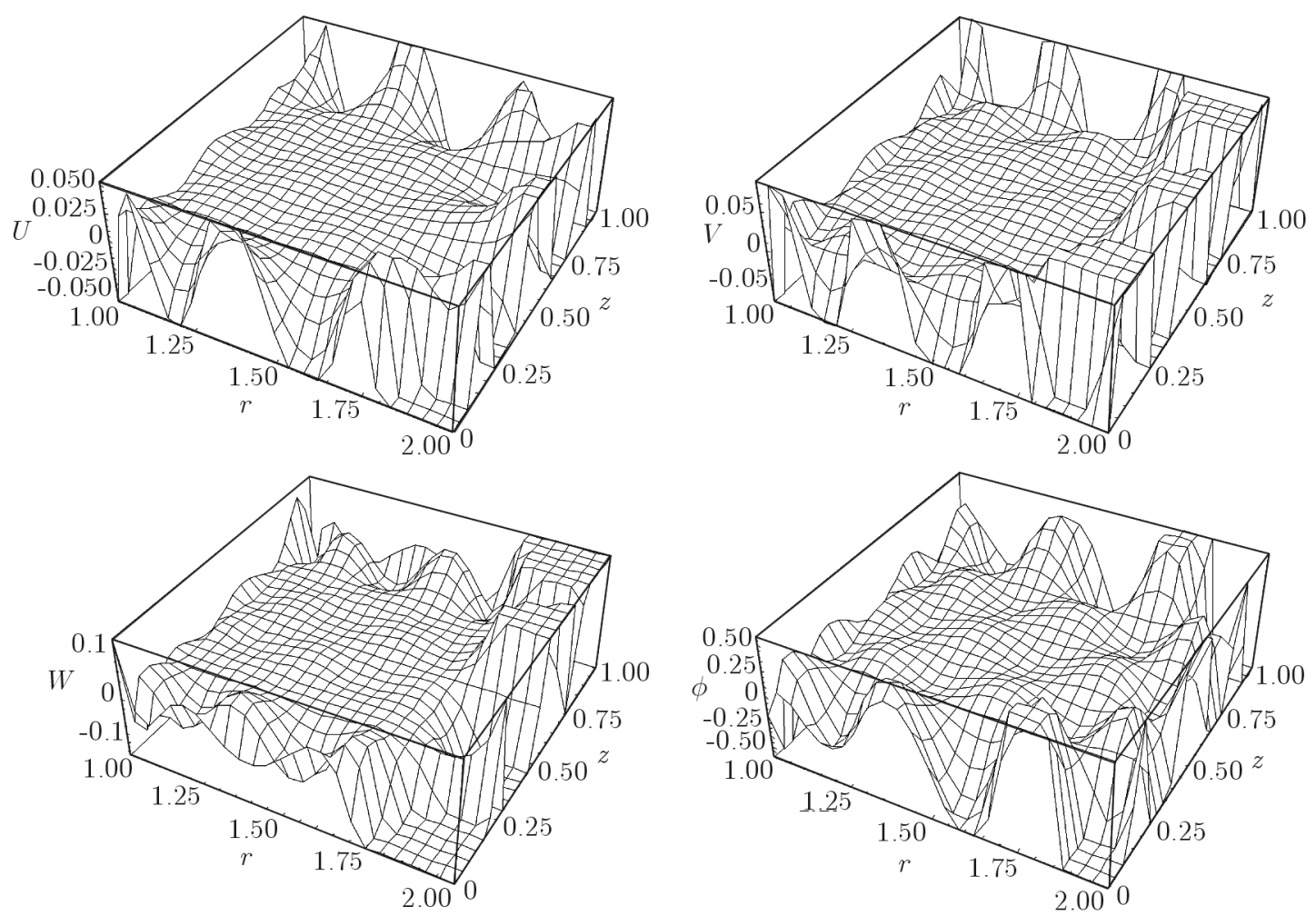

Fig. 8. Mechanical displacement and electric potential profiles of the first mode for a piezoelectric square ring with $\eta=2$ at $k d=4.1$ 
axial polarization. From the curves, we find that different radius to thickness ratios and width to height ratios and polarizing direction all have significant influence on the dispersion curves. The first two modes have no cut-off frequencies, which is different from that for an infinite hollow cylinder in which only the first mode has no cut-off frequencies. In an infinite hollow cylinder, only the thickness direction is a finite dimension, but there are two finite dimensions in a rectangular ring. With the width to height ratio increasing, the difference between the first mode dispersion curve and the second one becomes small, and the cut-off frequencies become small.

The displacement and electric potential profiles of the first mode for a square ring with $\eta=10$ at $k d=4.1$ and $k d=40.1$ are respectively shown in Figs. 7 and 8. We notice that displacement $u$ and $v$ and electric potential distributions are symmetry and displacement $w$ is antisymmetry in axial direction, This is because the geometry and material propreties are symmetric in axial direction. The displacement and electric potential profiles distribute mainly near the outside edge at small wavenumber case and distribute around the four boundaries at big wavenumber case.

\section{Conclusions and prospects}

The formulation to analyze the guided wave in piezoelectric rings with rectangular cross-sections using the extended orthogonal polynomial approach has been presented in this paper. According to the numerical results, we can draw the following conclusions:

(a) The effects of the piezoelectricity on dispersion curves become stronger with the wave number and mode order increasing.

(b) The width to height ratio, radius to thickness ratio and polarization all can significantly influence the guided wave characteristics in piezoelectric rings.

(c) The displacement and electric potential distributions are symmetry in axial direction and distribute mainly near the outside edge.

So, through changing the width to height ratio and the radius to thickness ratio of the piezoelectric ring, we can obtain the ring transducers with the dispersion features and field distributions that we want.

We consider that the present approach could be of interest in non-destructive testing evaluation, and can deal with $2 \mathrm{D}$ structures with more complex cross sections and multi-field coupled $2 \mathrm{D}$ structures.

\section{Acknowledgments}

The work is supported by the National Natural Science Foundation of China (No. 11272115) and Doctoral Fund of Henan Polytechnic University (No. B2009-81) and Foundation for Distinguished Young Scholars of Henan Polytechnic University (No. J2013-08) and by the high-performance grid computing platform of Henan Polytechnic University.

\section{References}

1. Cai C., Liu G.R., Lam K.Y., 2001, A technique for modelling multiple piezoelectric layers, Smart Materials And Structures, 10,689

2. Chakraborty A., 2009, Wave Propagation in Porous Piezoelectric Media, Computer Modeling in Engineering and Sciences, 40, 105

3. Han X., Liu G.R., Oнyoshi T., 2004, Dispersion and characteristic surfaces of waves in hybrid multilayered piezoelectric circular cylinders, Computational Mechanics, 33, 334-344 
4. Ing Y.S., LiaO H.F., Huang K.S., 2013, The extended Durbin method and its application for piezoelectric wave propagation problems, International Journal of Solids and Structures, 50, 4000-4009

5. Lefebvre J.E., Zhang V., Gazalet J., Gryba T., 1999, Legendre polynomial approach for modeling free-ultrasonic waves in multilayered plates, Journal of Applied Physics, 85, 3419-3427

6. Loveday P.W., 2006, Numerical comparison of patch and sandwich piezoelectric transducers for transmitting ultrasonic waves, Smart Structures and Materials. International Society for Optics and Photonics, 6166, 1-8

7. Paul H.S., Venkatesan M., 1987, Vibrations of a hollow circular cylinder of piezoelectric ceramics, The Journal of the Acoustical Society of America, 82, 952-956

8. Puzyrev V., Storozhev V., 2011, Wave propagation in axially polarized piezoelectric hollow cylinders of sector cross section, Journal of Sound and Vibration, 330, 4508-4518

9. Shul'Ga H.A., 2002, Propagation of harmonic waves in anisotropic piezoelectric cylinders. Homogeneous piezoceramic waveguides, International Applied Mechanics, 38, 933-953

10. Singh B.M., Rokne J., 2013, Propagation of SH waves in layered functionally gradient piezoelectric-piezomagnetic structures, Philosophical Magazine, 93, 1690-1700

11. Wilson L.O., Morrison J.A., 1977, Wave propagation in piezoelectric rods of hexagonal crystal symmetry, Journal of Mechanics and Applied Mathematics, 30, 387-395

12. Yu J.G., Lefebvre J.E., Guo Y.Q., 2013, Wave propagation in multilayered piezoelectric spherical plates, Acta Mechanica, 224, 1335-1349

13. Yu J.G., MA Q.J., 2008, Circumferential wave in functionally graded piezoelectric cylindrical curved plates, Acta Mechanica, 198, 171-190

14. Yu J.G., Wu B., Chen G.Q., 2009, Wave characteristics in functionally graded piezoelectric hollow cylinders, Archive of Applied Mechanics, 79, 807-824

15. Zenkour A.M., 2012, Piezoelectric behavior of an inhomogeneous hollow cylinder with thermal gradient, International Journal of Thermophysics, 33, 1288-1301 